\title{
Measurement of the branching ratio of $\bar{B}^{0} \rightarrow D^{*+} \tau^{-} \bar{\nu}_{\tau}$ relative to $\bar{B}^{0} \rightarrow D^{*+} \ell^{-} \bar{\nu}_{\ell}$ decays
} with a semileptonic tagging method

A. Abdesselam, ${ }^{87}$ I. Adachi, ${ }^{20,16}$ K. Adamczyk, ${ }^{63}$ H. Aihara, ${ }^{95}$ S. Al Said, ${ }^{87,39}$ K. Arinstein, ${ }^{5,67}$ Y. Arita, ${ }^{56}$ D. M. Asner, ${ }^{70}$ T. Aso, ${ }^{100}$ H. Atmacan, ${ }^{52}$ V. Aulchenko, $,{ }^{5}, 67$ T. Aushev,${ }^{55}$ R. Ayad,${ }^{87}$ T. Aziz, ${ }^{88}$ V. Babu, ${ }^{88}$ I. Badhrees,${ }^{87,38}$ S. Bahinipati, ${ }^{24}$ A. M. Bakich,${ }^{86}$ A. Bala,${ }^{71}$ Y. Ban,${ }^{72}$ V. Bansal,${ }^{70}$ E. Barberio, ${ }^{51}$ M. Barrett,,${ }^{19}$ W. Bartel, ${ }^{10}$ A. Bay ${ }^{44}$ I. Bedny, ${ }^{5,67}$ P. Behera,${ }^{26}$ M. Belhorn, ${ }^{9}$ K. Belous,${ }^{30}$ D. Besson,${ }^{54}$ V. Bhardwaj, ${ }^{84}$ B. Bhuyan, ${ }^{25}$ M. Bischofberger, ${ }^{59}$ J. Biswal, ${ }^{33}$ T. Bloomfield, ${ }^{51}$ S. Blyth,${ }^{61}$ A. Bobrov,${ }^{5,67}$ A. Bondar,${ }^{5,67}$ G. Bonvicini, ${ }^{103}$ C. Bookwalter ${ }^{70}$ C. Boulahouache, ${ }^{87}$ A. Bozek, ${ }^{63}$ M. Bračko, ${ }^{49,33}$ F. Breibeck, ${ }^{29}$ J. Brodzicka, ${ }^{63}$ T. E. Browder, ${ }^{19}$ D. Cervenkov, ${ }^{6}$ M.-C. Chang, ${ }^{12}$ P. Chang ${ }^{62}$ Y. Chao,${ }^{62}$ V. Chekelian, ${ }^{50}$ A. Chen,${ }^{60}$ K.-F. Chen, ${ }^{62}$ P. Chen, ${ }^{62}$ B. G. Cheon, ${ }^{18}$ K. Chilikin, ${ }^{45,}{ }^{54}$ R. Chistov, ${ }^{45,}{ }^{54}$ K. Cho, ${ }^{40}$ V. Chobanova, ${ }^{50}$ S.-K. Choi, ${ }^{17}$ Y. Choi, ${ }^{85}$

D. Cinabro, ${ }^{103}$ J. Crnkovic, ${ }^{23}$ J. Dalseno,${ }^{50,89}$ M. Danilov,${ }^{54,45}$ N. Dash,${ }^{24}$ S. Di Carlo, ${ }^{103}$ J. Dingfelder ${ }^{4}$

Z. Doležal ${ }^{6}$ Z. Drásal, ${ }^{6}$ A. Drutskoy ${ }^{45,}{ }^{54}$ S. Dubey, ${ }^{19}$ D. Dutta,${ }^{88}$ K. Dutta,${ }^{25}$ S. Eidelman, ${ }^{5,67}$ D. Epifanov,${ }^{95}$ S. Esen,${ }^{9}$ H. Farhat, ${ }^{103}$ J. E. Fast,${ }^{70}$ M. Feindt,${ }^{35}$ T. Ferber,${ }^{10}$ A. Frey, ${ }^{15}$ O. Frost,${ }^{10}$ M. Fujikawa, ${ }^{59}$ B. G. Fulsom, ${ }^{70}$ V. Gaur, ${ }^{88}$ N. Gabyshev, ${ }^{5,67}$ S. Ganguly, ${ }^{103}$ A. Garmash,,${ }^{5,67}$ D. Getzkow, ${ }^{13}$ R. Gillard, ${ }^{103}$ F. Giordano, ${ }^{23}$ R. Glattauer ${ }^{29}$ Y. M. Goh, ${ }^{18}$ P. Goldenzweig, ${ }^{35}$ B. Golob, ${ }^{46,33}$ D. Greenwald, ${ }^{90}$ M. Grosse Perdekamp, ${ }^{23,77}$ J. Grygier, ${ }^{35}$ O. Grzymkowska, ${ }^{63}$ H. Guo,${ }^{79}$ J. Haba, ${ }^{20,}{ }^{16}$ P. Hamer, ${ }^{15}$ Y. L. Han,${ }^{28}$ K. Hara, ${ }^{20}$ T. Hara, ${ }^{20,16}$ Y. Hasegawa, ${ }^{81}$ J. Hasenbusch, ${ }^{4}$ K. Hayasaka, ${ }^{65}$ H. Hayashii, ${ }^{59}$ X. H. He, ${ }^{72}$ M. Heck, ${ }^{35}$ M. T. Hedges ${ }^{19}$ D. Heffernan, ${ }^{69}$ M. Heider, ${ }^{35}$ A. Heller,${ }^{35}$ T. Higuchi, ${ }^{36}$ S. Himori,${ }^{93}$ S. Hirose,${ }^{56}$ T. Horiguchi,${ }^{93}$ Y. Hoshi,${ }^{92}$ K. Hoshina, ${ }^{98}$ W.-S. Hou, ${ }^{62}$ Y. B. Hsiung, ${ }^{62}$ C.-L. Hsu, ${ }^{51}$ M. Huschle, ${ }^{35}$ H. J. Hyun, ${ }^{43}$ Y. Igarashi, ${ }^{20}$ T. Iijima, ${ }^{57,56}$ M. Imamura, ${ }^{56} \mathrm{~K}$. Inami, ${ }^{56} \mathrm{G}$. Inguglia, ${ }^{10} \mathrm{~A}$. Ishikawa, ${ }^{93} \mathrm{~K}$. Itagaki, ${ }^{93} \mathrm{R}$. Itoh, ${ }^{20}{ }^{16} \mathrm{M}$. Iwabuchi, ${ }^{105} \mathrm{M}$. Iwasaki, ${ }^{95}$ Y. Iwasaki, ${ }^{20}$ S. Iwata, ${ }^{97}$ W. W. Jacobs,${ }^{27}$ I. Jaegle, ${ }^{19}$ H. B. Jeon, ${ }^{43}$ D. Joffe,${ }^{37}$ M. Jones,${ }^{19}$ K. K. Joo, ${ }^{8}$ T. Julius, ${ }^{51}$ H. Kakuno, ${ }^{97}$ J. H. Kang, ${ }^{105}$ K. H. Kang, ${ }^{43}$ P. Kapusta, ${ }^{63}$ S. U. Kataoka, ${ }^{58}$ E. Kato, ${ }^{93}$ Y. Kato, ${ }^{56}$ P. Katrenko, ${ }^{55}, 45$ H. Kawai, ${ }^{7}$ T. Kawasaki, ${ }^{65}$ T. Keck, ${ }^{35}$ H. Kichimi, ${ }^{20}$ C. Kiesling, ${ }^{50}$ B. H. Kim, ${ }^{80}$ D. Y. Kim, ${ }^{83}$ H. J. Kim, ${ }^{43}$ H.-J. Kim, ${ }^{105}$ J. B. Kim,${ }^{41}$ J. H. Kim,${ }^{40}$ K. T. Kim,${ }^{41}$ M. J. Kim, ${ }^{43}$ S. H. Kim,${ }^{18}$ S. K. Kim, ${ }^{80}$ Y. J. Kim, ${ }^{40}$ K. Kinoshita, ${ }^{9}$ C. Kleinwort, ${ }^{10}$ J. Klucar, ${ }^{33}$ B. R. Ko, ${ }^{41}$ N. Kobayashi, ${ }^{96}$ S. Koblitz, ${ }^{50}$ P. Kodyš, ${ }^{6}$ Y. Koga ${ }^{56}$ S. Korpar, ${ }^{49,33}$ D. Kotchetkov, ${ }^{19}$ R. T. Kouzes, ${ }^{70}$ P. Križan, ${ }^{46,33}$ P. Krokovny, ${ }^{5,67}$ B. Kronenbitter ${ }^{35}$ T. Kuhr, ${ }^{47}$ R. Kumar, ${ }^{74}$ T. Kumita,${ }^{97}$ E. Kurihara,${ }^{7}$ Y. Kuroki,${ }^{69}$ A. Kuzmin $,{ }^{5}, 67$ P. Kvasnička, ${ }^{6}$ Y.-J. Kwon, ${ }^{105}$ Y.-T. Lai, ${ }^{62}$ J. S. Lange, ${ }^{13}$ D. H. Lee, ${ }^{41}$ I. S. Lee, ${ }^{18}$ S.-H. Lee,${ }^{41}$ M. Leitgab, ${ }^{23,}{ }^{77}$ R. Leitner,${ }^{6}$ D. Levit, ${ }^{90}$ P. Lewis, ${ }^{19}$ C. H. Li ${ }^{51}$ H. Li, ${ }^{27}$ J. Li,${ }^{80}$ L. Li,${ }^{79}$ X. Li,${ }^{80}$ Y. Li,${ }^{102}$ L. Li Gioi,${ }^{50}$ J. Libby,${ }^{26}$ A. Limosani,${ }^{51}$ C. Liu,${ }^{79}$ Y. Liu,${ }^{9}$ Z. Q. Liu, ${ }^{28}$ D. Liventsev, ${ }^{102,20}$ A. Loos, ${ }^{84}$ R. Louvot, ${ }^{44}$ M. Lubej, ${ }^{33}$ P. Lukin, ${ }^{5,67}$ T. Luo, ${ }^{73}$ J. MacNaughton, ${ }^{20}$

M. Masuda ${ }^{94}$ T. Matsuda ${ }^{53}$ D. Matvienko, ${ }^{5,67}$ A. Matyja,${ }^{63}$ S. McOnie,${ }^{86}$ Y. Mikami, ${ }^{93}$ K. Miyabayashi, ${ }^{59}$ Y. Miyachi, ${ }^{104}$ H. Miyake, ${ }^{20,}{ }^{16}$ H. Miyata, ${ }^{65}$ Y. Miyazaki,${ }^{56}$ R. Mizuk,${ }^{45,}{ }^{54,55}$ G. B. Mohanty, ${ }^{88}$ S. Mohanty,${ }^{88,101}$ D. Mohapatra, ${ }^{70}$ A. Moll,,${ }^{50,89}$ H. K. Moon, ${ }^{41}$ T. Mori, ${ }^{56}$ T. Morii, ${ }^{36}$ H.-G. Moser, ${ }^{50}$ T. Müller, ${ }^{35}$ N. Muramatsu, ${ }^{75}$ R. Mussa,${ }^{32}$ T. Nagamine,${ }^{93}$ Y. Nagasaka, ${ }^{21}$ Y. Nakahama,${ }^{95}$ I. Nakamura $,{ }^{20},{ }^{16}$ K. R. Nakamura,${ }^{20}$ E. Nakano, ${ }^{68}$ H. Nakano, ${ }^{93}$ T. Nakano, ${ }^{76}$ M. Nakao, ${ }^{20,16}$ H. Nakayama, ${ }^{20,16}$ H. Nakazawa, ${ }^{60}$ T. Nanut, ${ }^{33}$ K. J. Nath,${ }^{25}$

Z. Natkaniec, ${ }^{63}$ M. Nayak, ${ }^{103}$ E. Nedelkovska, ${ }^{50}$ K. Negishi, ${ }^{93}$ K. Neichi, ${ }^{92}$ C. Ng, ${ }^{95}$ C. Niebuhr, ${ }^{10}$ M. Niiyama, ${ }^{42}$ N. K. Nisar ${ }^{88,1}$ S. Nishida, ${ }^{20,16}$ K. Nishimura, ${ }^{19}$ O. Nitoh, ${ }^{98}$ T. Nozaki,${ }^{20}$ A. Ogawa, ${ }^{77}$ S. Ogawa, ${ }^{91}$ T. Ohshima, ${ }^{56}$ S. Okuno, ${ }^{34}$ S. L. Olsen,${ }^{80}$ Y. Ono, ${ }^{93}$ Y. Onuki, ${ }^{95}$ W. Ostrowicz,${ }^{63}$ C. Oswald, ${ }^{4}$ H. Ozaki, ${ }^{20}, 16$ P. Pakhlov,${ }^{45,54}$ G. Pakhlova, ${ }^{45,}{ }^{55}$ B. Pal,${ }^{9}$ H. Palka, ${ }^{63}$ E. Panzenböck, ${ }^{15,}{ }^{59}$ C.-S. Park, ${ }^{105}$ C. W. Park,${ }^{85}$ H. Park,${ }^{43}$ K. S. Park, ${ }^{85}$ S. Paul, ${ }^{90}$ L. S. Peak, ${ }^{86}$ T. K. Pedlar, ${ }^{48}$ T. Peng, ${ }^{79}$ L. Pesántez, ${ }^{4}$ R. Pestotnik, ${ }^{33}$ M. Peters, ${ }^{19}$ M. Petrič,${ }^{33}$ L. E. Piilonen, ${ }^{102}$ A. Poluektov,${ }^{5,67}$ K. Prasanth,${ }^{26}$ M. Prim,${ }^{35}$ K. Prothmann, ${ }^{50,89}$ C. Pulvermacher, ${ }^{35}$ M. V. Purohit, ${ }^{84}$ J. Rauch,${ }^{90}$ B. Reisert ${ }^{50}$ E. Ribežl,${ }^{33}$ M. Ritter ${ }^{47}$ M. Röhrken, ${ }^{35}$ J. Rorie ${ }^{19}$ A. Rostomyan, ${ }^{10}$ M. Rozanska, ${ }^{63}$ S. Rummel, ${ }^{47}$ S. Ryu ${ }^{80}$ H. Sahoo, ${ }^{19}$ T. Saito, ${ }^{93}$ K. Sakai, ${ }^{20}$ Y. Sakai, ${ }^{20}, 16$ S. Sandilya,${ }^{9}$ D. Santel, ${ }^{9}$ L. Santelj, ${ }^{20}$ T. Sanuki,${ }^{93}$ N. Sasao, ${ }^{42}$ Y. Sato, ${ }^{57}$ V. Savinov,${ }^{73}$ T. Schlüter,${ }^{47}$ O. Schneider, ${ }^{44}$ G. Schnell,,${ }^{2,22}$ P. Schönmeier, ${ }^{93}$ M. Schram, ${ }^{70}$ C. Schwanda, ${ }^{29}$ A. J. Schwartz, ${ }^{9}$ B. Schwenker, ${ }^{15}$ R. Seidl, ${ }^{77}$ Y. Seino, ${ }^{65}$ A. Sekiya, ${ }^{59}$ D. Semmler, ${ }^{13}$ K. Senyo, ${ }^{104}$ O. Seon, ${ }^{56}$ I. S. Seong, ${ }^{19}$ M. E. Sevior, ${ }^{51}$ L. Shang, ${ }^{28}$ M. Shapkin, ${ }^{30}$ V. Shebalin, ${ }^{5,} 67$ C. P. Shen, ${ }^{3}$ T.-A. Shibata ${ }^{96}$ H. Shibuya, ${ }^{91}$ S. Shinomiya, ${ }^{69}$ J.-G. Shiu, ${ }^{62}$ B. Shwartz, ${ }^{5,67}$ A. Sibidanov ${ }^{86}$ F. Simon, ${ }^{50,89}$ J. B. Singh, ${ }^{71}$ R. Sinha ${ }^{31}$ P. Smerkol, ${ }^{33}$ Y.-S. Sohn, ${ }^{105}$ A. Sokolov, ${ }^{30}$ Y. Soloviev, ${ }^{10}$ E. Solovieva, ${ }^{45,55}$ S. Stanič ${ }^{66}$ M. Starič ${ }^{33}$ M. Steder, ${ }^{10}$ J. F. Strube, ${ }^{70}$ J. Stypula, ${ }^{63}$ S. Sugihara,${ }^{95}$ A. Sugiyama, ${ }^{78}$ M. Sumihama, ${ }^{14}$ K. Sumisawa, ${ }^{20,16}$ T. Sumiyoshi, ${ }^{97}$ K. Suzuki, ${ }^{56}$ S. Suzuki, ${ }^{78}$ S. Y. Suzuki, ${ }^{20}$

Z. Suzuki,${ }^{93}$ H. Takeichi,,${ }^{56}$ M. Takizawa, ${ }^{82}$ U. Tamponi, ${ }^{32,}{ }^{99}$ M. Tanaka, ${ }^{20,}{ }^{16}$ S. Tanaka, ${ }^{20}, 16$ K. Tanida, ${ }^{80}$ N. Taniguchi, ${ }^{20}$ G. N. Taylor, ${ }^{51}$ Y. Teramoto, ${ }^{68}$ I. Tikhomirov, ${ }^{54}$ K. Trabelsi, ${ }^{20,}{ }^{16}$ V. Trusov ${ }^{35}$ Y. F. Tse ${ }^{51}$ 
T. Tsuboyama, ${ }^{20,16}$ M. Uchida, ${ }^{96}$ T. Uchida, ${ }^{20}$ S. Uehara, ${ }^{20,16}$ K. Ueno, ${ }^{62}$ T. Uglov, ${ }^{45,}{ }^{55}$ Y. Unno, ${ }^{18} \mathrm{~S}$. Uno, ${ }^{20,16}$ S. Uozumi, ${ }^{43}$ P. Urquijo, ${ }^{51}$ Y. Ushiroda, ${ }^{20,16}$ Y. Usov, ${ }^{5,67}$ S. E. Vahsen, ${ }^{19}$ C. Van Hulse, ${ }^{2}$ P. Vanhoefer, ${ }^{50}$ G. Varner, ${ }^{19}$ K. E. Varvell, ${ }^{86}$ K. Vervink, ${ }^{44}$ A. Vinokurova, ${ }^{5,67}$ V. Vorobyev, ${ }^{5,67}$ A. Vossen, ${ }^{27}$ M. N. Wagner, ${ }^{13}$ C. H. Wang, ${ }^{61}$ J. Wang, ${ }^{72}$ M.-Z. Wang, ${ }^{62}$ P. Wang, ${ }^{28}$ X. L. Wang, ${ }^{102}$ M. Watanabe, ${ }^{65}$ Y. Watanabe, ${ }^{34}$ R. Wedd, ${ }^{51}$ S. Wehle ${ }^{10}$ E. White, ${ }^{9}$ J. Wiechczynski, ${ }^{63}$ K. M. Williams, ${ }^{102}$ E. Won, ${ }^{41}$ B. D. Yabsley, ${ }^{86}$ S. Yamada, ${ }^{20}$ H. Yamamoto, ${ }^{93}$ J. Yamaoka, ${ }^{70}$ Y. Yamashita, ${ }^{64}$ M. Yamauchi, ${ }^{20,}{ }^{16}$ S. Yashchenko, ${ }^{10}$ H. Ye, ${ }^{10}$ J. Yelton, ${ }^{11}$ Y. Yook, ${ }^{105}$ C. Z. Yuan, ${ }^{28}$ Y. Yusa, ${ }^{65}$ C. C. Zhang, ${ }^{28}$ L. M. Zhang, ${ }^{79}$ Z. P. Zhang, ${ }^{79}$ L. Zhao, ${ }^{79}$ V. Zhilich, ${ }^{5,67}$ V. Zhukova, ${ }^{54}$ V. Zhulanov, ${ }^{5,67}$ M. Ziegler, ${ }^{35}$ T. Zivko, ${ }^{33}$ A. Zupanc, ${ }^{46,}{ }^{33}$ N. Zwahlen, ${ }^{44}$ and O. Zyukova ${ }^{5,67}$

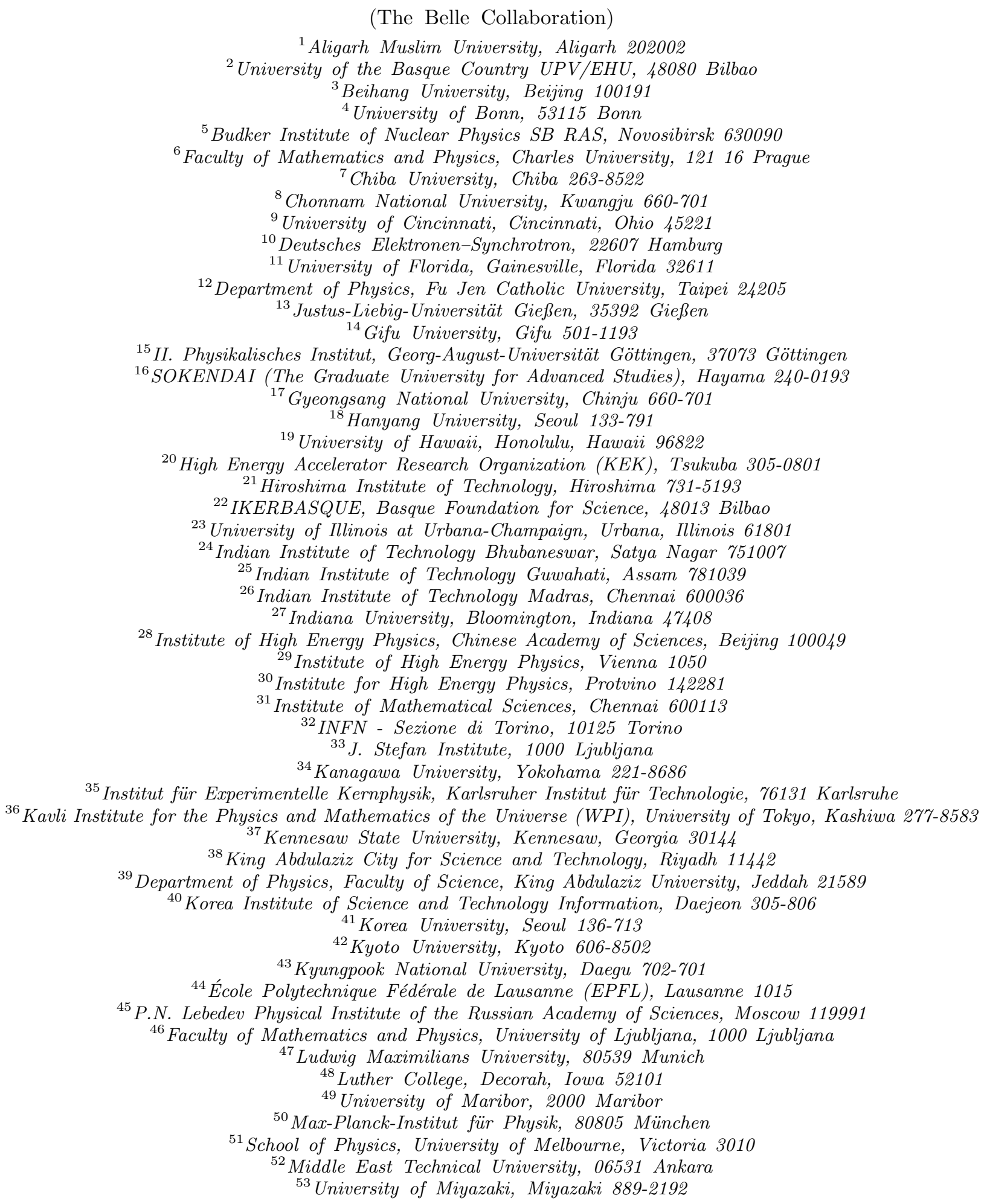




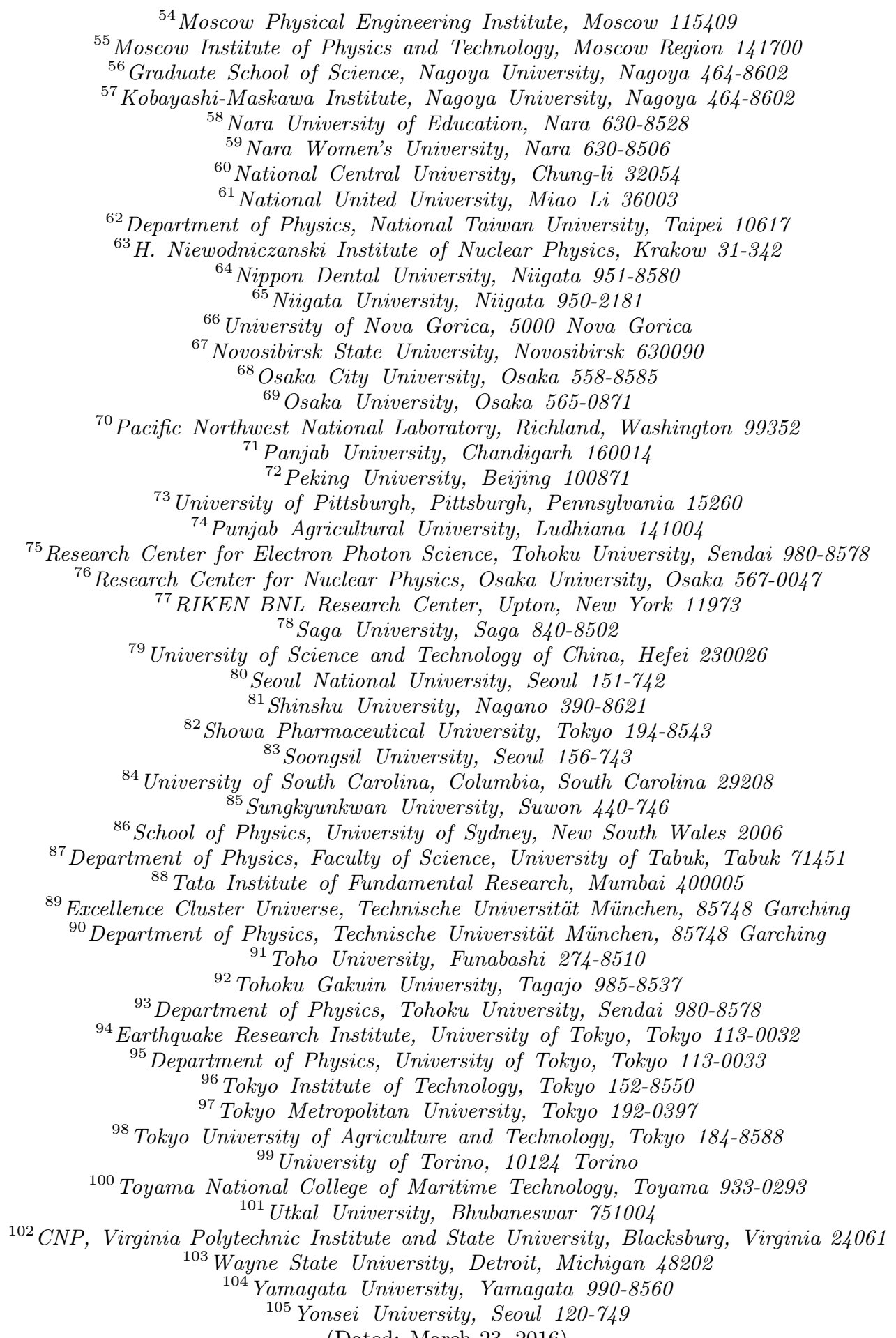

(Dated: March 23, 2016)

We report a measurement of ratio $\mathcal{R}\left(D^{*}\right)=\mathcal{B}\left(\bar{B}^{0} \rightarrow D^{*+} \tau^{-} \bar{\nu}_{\tau}\right) / \mathcal{B}\left(\bar{B}^{0} \rightarrow D^{*+} \ell^{-} \bar{\nu}_{\ell}\right)$, where $\ell$ denotes an electron or a muon. The results are based on a data sample containing $772 \times 10^{6} B \bar{B}$ pairs recorded at the $\Upsilon(4 S)$ resonance with the Belle detector at the KEKB $e^{+} e^{-}$collider. We select a sample of $B^{0} \bar{B}^{0}$ pairs by reconstructing both $B$ mesons in semileptonic decays to $D^{* \mp} \ell^{ \pm}$. We measure $\mathcal{R}\left(D^{*}\right)=0.302 \pm 0.030$ (stat) \pm 0.011 (syst), which is within $1.6 \sigma$ of the Standard Model theoretical expectation, where $\sigma$ is the standard deviation including systematic uncertainties.

\section{INTRODUCTION}

Semitauonic $B$ meson decays of the type $b \rightarrow c \tau \nu_{\tau}[1]$ are sensitive probes to search for physics beyond the
Standard Model (SM). Charged Higgs bosons, which appear in supersymmetry and other models with at least 
two Higgs doublets, may contribute to the decay to due to large mass of the $\tau$ lepton and induce measurable effects in the branching fraction. Similarly, leptoquarks, which carry both baryon number and lepton number, may also contribute to this process. The ratio of branching fractions

$$
\mathcal{R}\left(D^{(*)}\right)=\frac{\mathcal{B}\left(\bar{B} \rightarrow D^{(*)} \tau^{-} \bar{\nu}_{\tau}\right)}{\mathcal{B}\left(\bar{B} \rightarrow D^{(*)} \ell^{-} \bar{\nu}_{\ell}\right)} \quad(\ell=e, \mu),
$$

is typically used instead of the absolute branching fraction of $\bar{B} \rightarrow D^{(*)+} \tau^{-} \bar{\nu}_{\tau}$, to reduce several systematic uncertainties such as those on the experimental efficiency, the CKM matrix elements $\left|V_{c b}\right|$, and on the form factors. The SM calculations on these ratios predict $\mathcal{R}\left(D^{*}\right)=0.252 \pm 0.003[2]$ and $\mathcal{R}(D)=0.297 \pm 0.017$ [3, 7] with precision of better than $2 \%$ and $6 \%$ for $\mathcal{R}\left(D^{*}\right)$ and $\mathcal{R}(D)$, respectively. Exclusive semitauonic $B$ decays were first observed by the Belle Collaboration [5], with subsequent studies reported by Belle [6, 7], BABAR [4], and LHCb 8] Collaborations. All results are consistent with each other, and the average values of Refs. [4, 7, 8] have been found to be $\mathcal{R}\left(D^{*}\right)=0.322 \pm 0.018 \pm 0.012$ and $\mathcal{R}(D)=0.391 \pm 0.041 \pm 0.028$ [9], which exceed the SM predictions for $\mathcal{R}\left(D^{*}\right)$ and $\mathcal{R}(D)$ by $3.0 \sigma$ and $1.7 \sigma$, respectively. The combined analysis of $\mathcal{R}\left(D^{*}\right)$ and $\mathcal{R}(D)$, taking into account measurement correlations, finds that the deviation is $3.9 \sigma$ from the SM prediction.

So far, measurements of $\mathcal{R}\left(D^{(*)}\right)$ at the $B$ factories have been performed either using a hadronic [4, 7] or an inclusive tagging method [5, 6]. Semileptonic tagging methods have been employed for use in studies of $B^{-} \rightarrow \tau^{-} \bar{\nu}_{\tau}$ decays, and have been shown to be of similar experimental precision to that of the hadronic tagging method [10, 11]. In this paper, we report the first measurement of $\mathcal{R}\left(D^{*}\right)$ using the semileptonic tagging method. We reconstruct signal $B^{0} \bar{B}^{0}$ events in modes where one $B$ decays semi-tauonically $\bar{B}^{0} \rightarrow D^{*+} \tau^{-} \bar{\nu}_{\tau}$ where $\tau^{-} \rightarrow \ell^{-} \bar{\nu}_{\ell} \nu_{\tau}$, (referred to hereafter as $B_{\text {sig }}$ ) and the the other $B$ decays in a semileptonic channel $\bar{B}^{0} \rightarrow D^{*+} \ell^{-} \bar{\nu}_{\ell}$ (referred to hereafter as $B_{\text {tag }}$ ). To reconstruct normalization $B^{0} \bar{B}^{0}$ events, which correspond to the denominator in $\mathcal{R}\left(D^{*}\right)$, we use both $B$ mesons decaying to semileptonic decay modes $D^{* \pm} \ell^{\mp} \widehat{\nu}_{\ell}$.

\section{DETECTOR AND MC SIMULATION}

We use the full $\Upsilon(4 S)$ data sample containing $772 \times 10^{6}$ $B \bar{B}$ pairs recorded with the Belle detector [12] at the $\mathrm{KEKB} e^{+} e^{-}$collider [13]. The Belle detector is a generalpurpose magnetic spectrometer which consists of a silicon vertex detector (SVD), a 50-layer central drift chamber (CDC), an array of aerogel threshold Cherenkov counters (ACC), time-of-flight scintillation counters (TOF), and an electromagnetic calorimeter (ECL) comprised of CsI(Tl) crystals. The devices are located inside a superconducting solenoid coil that provides a $1.5 \mathrm{~T}$ magnetic field. An iron flux-return located outside the coil is instrumented to detect $K_{L}^{0}$ mesons and to identify muons (KLM). The detector is described in detail elsewhere [12].

To determine the acceptance and probability density functions (PDF) for signal $\bar{B}^{0} \rightarrow D^{*+} \tau^{-} \bar{\nu}_{\tau}$, normalization $\bar{B}^{0} \rightarrow D^{*+} \ell^{-} \bar{\nu}_{\ell}$, and background processes we use Monte Carlo (MC) simulated events, which are based on the EvtGen event generator [14] and the GEANT3 package [15]. The MC samples for signal $\bar{B}^{0} \rightarrow D^{*+} \tau^{-} \bar{\nu}_{\tau}$ events are generated using the decay model based on the heavy quark effective theory [16].

Background $B \rightarrow D^{* *} \ell \nu_{\ell}$ events are simulated with the ISGW [17] model and reweighted to match the kinematics predicted by the LLSW model [18]. Here, $D^{* *}$ denotes the orbitally excited states, $D_{1}, D_{2}^{*}, D_{1}^{\prime}$, and $D_{0}^{*}$. Radially excited states are considered negligible. The normalization mode $B \rightarrow D^{*} \ell \nu_{\ell}$ is simulated using HQET, and reweighted according to the current world average form factor values: $\rho^{2}=1.207 \pm 0.015 \pm 0.021, R_{1}=$ $1.403 \pm 0.033$, and $R_{2}=0.854 \pm 0.020$ [9]. The sample sizes of the signal, $B \bar{B}$, and continuum $q \bar{q}(q=u, d, s, c)$ production processes correspond to about 40,10 and 6 times the integrated luminosity of the on-resonance collision data sample, respectively.

\section{EVENT SELECTION}

Charged particle tracks are reconstructed with the SVD and CDC, and the tracks other than $K_{S}^{0} \rightarrow \pi^{+} \pi^{-}$ daughters are required to originate from near the interaction region. Electrons are identified by a combination of the specific ionization $(d E / d x)$ in the $\mathrm{CDC}$, the ratio of the cluster energy in the ECL to the track momentum measured with the SVD and CDC, the response of the ACC, the shower shape in the ECL, and the match between the positions of the shower and the track at the ECL surface. To recover bremsstrahlung photons from electrons, we add the four-momentum of each photon detected within 0.05 radians of the original track direction. Muons are identified by the track penetration depth and hit distribution in the KLM. Charged kaons are identified by combining information from the $d E / d x$ in the $\mathrm{CDC}$, the flight time measured with the TOF, and the response of the ACC [19]. We do not apply any particle identification criteria on charged pions.

Candidate $K_{S}^{0}$ mesons are formed by combining two oppositely charged tracks with pion mass hypotheses. We require the invariant mass to lie within $15 \mathrm{MeV} / c^{2}$ of the nominal $K^{0}$ mass [20]. We then impose the following additional requirements: (1) the two pion tracks must have a large distance of closest approach to the IP in the plane perpendicular to the electron beam line; (2) the pion tracks must intersect at a common vertex that is displaced from the IP; (3) the $K_{S}^{0}$ candidate's momentum vector should originate from the IP. Neutral pion candidates are formed from pairs of photons with further criteria specific to whether the $\pi^{0}$ is from a $D^{*+}$ decay 
and $D$ decay. For the neutral pions from $D$ decays, we require the photon daughter energies to be greater than $50 \mathrm{MeV}$, the cosine of the angle between two photons to be greater than 0.0 , and the $\gamma \gamma$ invariant mass to be -15 to $+10 \mathrm{MeV} / c^{2}$ around the nominal $\pi^{0}$ mass [20] which corresponds to approximately $\pm 1.8 \sigma$, where photons are measured as an energy cluster in the ECL with no associated charged tracks. A mass-constrained fit is then performed to obtain the $\pi^{0}$ momentum. For neutral pions from $D^{*+}$ decays, which have lower energies, we require one photon to have at least $50 \mathrm{MeV}$ and the other to have at least $20 \mathrm{MeV}$. We apply a tighter window on the invariant mass to compensate for the lower photon energy requirement, within $10 \mathrm{MeV} / c^{2}$ of the nominal $\pi^{0}$ mass, which corresponds to approximately $\pm 1.6 \sigma$.

Neutral $D$ mesons are reconstructed in the following decay modes: $D^{0} \rightarrow K^{-} \pi^{+}, K_{S}^{0} \pi^{0}, K^{+} K^{-}, \pi^{+} \pi^{-}$, $K_{S}^{0} \pi^{+} \pi^{-}, K^{-} \pi^{+} \pi^{0}, \pi^{+} \pi^{-} \pi^{0}, K_{S}^{0} K^{+} K^{-}, K^{-} \pi^{+} \pi^{+} \pi^{-}$, and $K_{S}^{0} \pi^{+} \pi^{-} \pi^{0}$. Charged $D$ mesons are reconstructed in the following modes: $D^{+} \rightarrow K_{S}^{0} \pi^{+}, K^{-} \pi^{+} \pi^{+}$, $K_{S}^{0} \pi^{+} \pi^{0}, K^{+} K^{-} \pi^{+}$, and $K_{S}^{0} \pi^{+} \pi^{+} \pi^{-}$. The combined reconstructed branching fractions are $37 \%$ and $22 \%$ for $D^{0}$ and $D^{+}$, respectively. For $D$ decay modes without a $\pi^{0}$ in the final state, we require the invariant mass of the $D$ candidates to be within $15 \mathrm{MeV} / c^{2}$ of the $D^{0}$ or $D^{+}$ mass, which corresponds to a window of approximately $\pm 3 \sigma$. For modes with a $\pi^{0}$ in the final state, we require a wider invariant mass window: from -45 to $+30 \mathrm{MeV} / c^{2}$ around the nominal $D^{0}$ mass for $D^{0}$ candidates and from -36 to $+24 \mathrm{MeV} / c^{2}$ around the nominal $D^{+}$mass for $D^{+}$candidates. Candidate $D^{*+}$ mesons are formed by combining $D^{0}$ and $\pi^{+}$candidates or $D^{+}$and $\pi^{0}$ candidates. To improve the resolution of the $D^{*}-D$ mass difference, $\Delta M$, the charged pion track from the $D^{*+}$ is refitted to the $D^{0}$ decay vertex. We require $\Delta M$ to be within $2.5 \mathrm{MeV} / c^{2}$ and $2.0 \mathrm{MeV} / c^{2}$ around nominal $D^{*}$ $D$ mass difference for $D^{*+} \rightarrow D^{0} \pi^{+}$and $D^{*+} \rightarrow D^{+} \pi^{0}$ decay modes, respectively. We apply a tighter window in $D^{*+} \rightarrow D^{+} \pi^{0}$ decay mode to suppress large background from fake neutral pions.

To tag semileptonic $B$ decays, we combine $D^{*+}$ meson and lepton candidates of opposite electric charge and calculate the cosine of the angle between the momentum of the $B$ meson and the $D^{*} \ell$ system in the $\Upsilon(4 S)$ rest frame, under the assumption that only one massless particle is not reconstructed:

$$
\cos \theta_{B-D^{*} \ell} \equiv \frac{2 E_{\mathrm{beam}} E_{D^{*} \ell}-m_{B}^{2}-M_{D^{*} \ell}^{2}}{2\left|\vec{p}_{B}\right| \cdot\left|\vec{p}_{D^{*} \ell}\right|},
$$

where $E_{\text {beam }}$ is the energy of the beam, and $E_{D^{*} \ell}, \vec{p}_{D^{*} \ell}$ and $M_{D^{*} \ell}$ are the energy, momentum, and mass of the $D^{*} \ell$ system, respectively. The variable $m_{B}$ is the nominal $B$ meson mass [20], and $\vec{p}_{B}$ is the nominal $B$ meson momentum. All variables are defined in the $\Upsilon(4 S)$ rest frame. Correctly reconstructed $B$ candidates in the tag and normalization mode $D^{*} \ell \nu_{\ell}$ are expected to have a value of $\cos \theta_{B-D^{*} \ell}$ between -1 and +1 . On the other hand, correctly reconstructed $B$ candidates in the sig- nal decay mode $D^{*} \tau \nu_{\tau}$ or falsely reconstructed $B$ candidates would tend to have values of $\cos \theta_{B-D^{*} \ell}$ below the physical region due to contributions from additional particles and a large negative correlation with missing mass squared, $M_{\text {miss }}^{2}=\left(2 E_{\text {beam }}-\sum_{i} E_{i}\right)^{2} / c^{4}-\left|\sum_{i} \vec{p}_{i}\right|^{2} / c^{2}$, where $\left(\vec{p}_{i}, E_{i}\right)$ is four-momentum of the particles in the $\Upsilon(4 S)$ rest frame.

In each event we require two tagged $B$ candidates that are opposite in flavor. Signal events may have the same flavor due to the $B \bar{B}$ mixing, however we veto such events as they lead to ambiguous $D^{*} \ell$ pair assignment and larger combinatorial background. We require that at most one $B$ meson is reconstructed in a $D^{+}$mode, in order to avoid large background from fake neutral pions when forming $D^{*}$ candidates. In each signal event we assign the candidate with the lowest value of $\cos \theta_{B-D^{*} \ell}$ (referred to hereafter as $\left.\cos \theta_{B-D^{*} \ell}^{\mathrm{sig}}\right)$ as $B_{\text {sig }}$. The probability of falsely assigning the $B_{\text {sig }}$ as the $B_{\text {tag }}$ for signal events is about $3 \%$. After the identification of the $B_{\text {sig }}$ and $B_{\text {tag }}$ candidates, we apply further background suppression criteria. On the tag side $\left(B_{\text {tag }}\right)$ we require $-2.0<\cos \theta_{B-D^{*} \ell}^{\text {tag }}<+1.5$ in order to select $B \rightarrow D^{*} \ell \nu_{\ell}$. On the signal side we require the $D^{*}$ momentum in the $\Upsilon(4 S)$ rest frame to be less than $2.0 \mathrm{GeV} / c$, while we require it to be less than 2.5 $\mathrm{GeV} / c$ on the tag side, which accounts for differing lepton masses. Finally, we require the events to contain no extra charged tracks, $K_{S}^{0}$ candidates, or $\pi^{0}$ candidates, which are reconstructed with the same criteria as those used in the $D$ candidates. At this stage, the probability of finding multiple candidates is $7 \%$, and the average number of candidates is 1.08 . When multiple candidates are found in an event, we select the most signal-like events based on the quality of vertex-constrained fits for the $D$ mesons.

\section{BACKGROUND SUPPRESSION}

To separate reconstructed signal and normalization events, we employ a neural network approach based on the "NeuroBayes" software package [21]. The variables used as inputs to the network are (i) $\cos \theta_{B-D^{*} \ell}^{\operatorname{sig}}$, (ii) missing mass squared, $M_{\text {miss }}^{2}$, and (iii) visible energy $E_{\mathrm{vis}}=\sum_{i} E_{i}$, where $E_{i}$ is energies of the particles in the $\Upsilon(4 S)$ rest frame. The most powerful observable in separating signal and background is $\cos \theta_{B-D^{*} \ell}^{\text {sig }}$. The neural network is trained using $\mathrm{MC}$ samples of signal and normalization events.

The most dominant background contribution arises from events with falsely reconstructed (fake) $D^{(*)}$ mesons. We categorize events, in which $D^{(*)}$ candidates are falsely reconstructed in any events, into fake $D^{(*)}$ events. The next most dominant contributions arise from two sources in which $D^{*}$ mesons from both $B_{\text {sig }}$ and $B_{\text {tag }}$ are correctly reconstructed. One source is $B \rightarrow D^{* *} \ell \nu_{\ell}$, where the $D^{* *}$ decays to $D^{(*)}$ along with accompanying particles. The other source is $B \rightarrow X_{c} D^{*}$ events, where one $D^{*}$ meson is correctly reconstructed and the other charmed meson $X_{c}$ decays via a semileptonic mode. If 
the hadrons in the semileptonic $X_{c}$ decay are not identified, such events can mimic signal. Similarly, when $X_{c}$ is $D_{s}^{+}$meson which decays into $\tau^{+} \nu_{\tau}$, such events can also mimic signal. To separate signal and normalization events from background processes, we use the extra energy, $E_{\mathrm{ECL}}$, which is defined as the sum of the energies of neutral clusters detected in the ECL that are not associated with reconstructed particles. To mitigate photons related to beam background in the energy sum, we only include clusters with energies greater than 50, 100, and $150 \mathrm{MeV}$ for the barrel, forward, and backward calorimeter regions, respectively. Signal and normalization events peak near zero in $E_{\mathrm{ECL}}$, while backgrounds populate a wider range. We require $E_{\mathrm{ECL}}$ to be less than $1.2 \mathrm{GeV}$.

\section{MC CALIBRATION}

To improve the accuracy of the MC simulation we apply a series of calibration factors determined from control sample measurements. The lepton identification efficiencies are corrected for electrons and muons, respectively, to account for differences between the detector responses in data and MC. We reweight events to account for differing $D^{(*)}$ yields between data and MC samples. The differing yields of truly reconstructed $D^{(*)}$ mesons between data and $\mathrm{MC}$ samples affect $\mathcal{R}\left(D^{*}\right)$ measurements through the determination of the backgrounds. It is difficult to precisely estimate the differing yields of falsely reconstructed $D^{(*)}$ mesons between data and MC samples by only using sideband region in two-dimensional of the $D$ invariant masses $\left(M_{D}\right)$ or $\Delta M$. Therefore, calibration factors for events with both correctly and falsely reconstructed $D$ mesons are estimated for each $D$ meson subdecay mode using a two-dimensional fit to $M_{D}$. Precise calibration can be performed by using samples with two tagged candidates, which have good purity and are close to final samples for the $\mathcal{R}\left(D^{*}\right)$ measurement. A twodimensional PDF is constructed by taking the product of the one-dimensional functions for $M_{D}$. The function in each dimension is constructed by the sum of the signal component and the background component as modeled by first-order Chebychev polynomials. The signal component is modeled by a triple Gaussian for $D^{0}$ decay modes without a $\pi^{0}$ or a Crystal Ball function 22] plus a Gaussian for $D^{0}$ decay modes with a $\pi^{0}$ and $D^{+}$decay modes. In this calibration, we do not distinguish signal and tag side. To estimate calibration factors for specific $D$ subdecay modes, we fit samples in which one $D$ meson is reconstructed in a specific mode while the other $D$ meson is reconstructed in any signal mode. From the signal and background yield ratios of data to MC samples, we derive calibration factors of the specific sub-decay mode for events with correctly and falsely reconstructed $D$ mesons. We can not independently determine calibration factors for all $D$ meson sub-decay modes, as we use other subdecay modes when we calibrate one specific sub-decay mode of a given $D$ meson. To estimate all the calibration factors correctly, we first perform the two-dimensional fitting for each sub-decay mode separately, then repeat the process, weighting samples by the estimated calibration factors, until all calibration factors converge. Similarly, we estimate calibration factors for events with correctly and falsely reconstructed $D^{*}$ mesons from a twodimensional fit to $\Delta M$. Calibration factors for events with correctly and falsely reconstructed $D^{*}$ mesons are separately estimated for $D^{0}$ and $D^{+}$mesons.

\section{MAXIMUM LIKELIHOOD FIT}

We extract the signal and normalization yields using a two-dimensional extended maximum-likelihood fit in $N N$ and $E_{\mathrm{ECL}}$. The likelihood function consists of five components: signal, normalization, fake $D^{(*)}$ events, $B \rightarrow D^{* *} \ell \nu_{\ell}$, and other backgrounds predominantly from $B \rightarrow X_{c} D^{*}$. The PDFs of all components are determined based on MC simulation. There are significant correlations between $N N$ and $E_{\mathrm{ECL}}$ in the background components, but not for the signal. We therefore construct the background PDFs using two-dimensional histogram PDFs, and apply a smoothing procedure to account for limited statistical power [23]. We construct the signal PDF by taking the product of one-dimensional histograms in $N N$ and $E_{\mathrm{ECL}}$.

Three parameters are floated in the final fit, corresponding to the yields of the signal, normalization, and $B \rightarrow D^{* *} \ell \nu_{\ell}$ components. The yields of fake $D^{(*)}$ events are fixed to the values estimated from sidebands in the $\Delta M$ distributions. Since the PDF shape of fake $D^{(*)}$ events depends on the composition of signal, normalization, $B \rightarrow D^{* *} \ell \nu_{\ell}$, and other backgrounds, the relative contributions of these processes to the fake $D^{(*)}$ component are described as a function of the the three fitting parameters. The yields of other backgrounds are fixed to the values expected from MC simulation. The ratio $\mathcal{R}\left(D^{*}\right)$ is derived from the formula:

$$
\mathcal{R}\left(D^{*}\right)=\frac{1}{2 \mathcal{B}\left(\tau^{-} \rightarrow \ell^{-} \bar{\nu}_{\ell} \nu_{\tau}\right)} \cdot \frac{\varepsilon_{\mathrm{norm}}}{\varepsilon_{\mathrm{sig}}} \cdot \frac{N_{\mathrm{sig}}}{N_{\mathrm{norm}}},
$$

where $\varepsilon_{\operatorname{sig}(\text { norm })}$ and $N_{\operatorname{sig}(\text { norm) }}$ are reconstruction efficiency and yields of signal (normalization) events. The branching ratios of $\tau^{-} \rightarrow \ell^{-} \bar{\nu}_{\ell} \nu_{\tau}$ are based on the current world average values [20]. The ratio of efficiencies, $\varepsilon_{\text {norm }} / \varepsilon_{\text {sig }}$, is estimated to be $1.289 \pm 0.015$ from MC simulation. The difference between reconstruction efficiencies of signal and normalization events arises from their distinct lepton momentum distributions, and the differing event criterion on the $D^{*}$ momenta on the signal side.

We validate the PDFs used in the fitting procedure by analysing various control samples. For fake $D^{(*)}$ events we study the $\Delta M$ sidebands, where we find good agreement in both $N N$ and $E_{\mathrm{ECL}}$. For $B \rightarrow D^{*} \ell \nu_{\ell}$ decays, we require one $B$ meson to be reconstructed with the hadronic tagging method, and the other $B$ meson reconstructed with the nominal criteria of this analysis. We 
find good agreement between data and $\mathrm{MC}$ in the $E_{\mathrm{ECL}}$, $M_{\text {miss }}^{2}$, and $E_{\text {vis }}$ distributions, while we find small discrepancies in the $\cos \theta_{B-D^{*} \ell}$ distributions and thus include the differences as a systematic uncertainty.

\section{SYSTEMATIC UNCERTAINTIES}

To estimate the systematic uncertainties on $\mathcal{R}\left(D^{*}\right)$, we vary all assumed parameters by one standard deviation and repeat the fit taking the resulting change in $\mathcal{R}\left(D^{*}\right)$. The systematic uncertainties are summarized in Table I. The dominant systematic uncertainty arises from the limited size of the MC samples: to estimate this uncertainty, we recalculated PDFs for signal, normalization, fake $D^{(*)}$ events, $B \rightarrow D^{* *} \ell \nu_{\ell}$, and other backgrounds by generating toy MC samples from the nominal PDFs according to Poisson statistics and repeated the fit with the new PDFs. Small discrepancies between the data and $\mathrm{MC}$ are found in the $\cos \theta_{B-D^{*} \ell}$ distributions in the hadronic tagged samples. We correct the $\cos \theta_{B-D^{*} \ell}$ distribution in MC samples according to the observed discrepancy and repeat the fit. The estimated uncertainty are referred as "PDF shape of the normalization in $\cos \theta_{B-D^{*}} \ell^{\prime}$ in Table I. The branching ratios of the $B \rightarrow D^{* *} \ell \nu_{\ell}$ decay modes and the decays of the $D^{* *}$ mesons are not well known, and therefore they contribute a large uncertainty in PDF shape of $B \rightarrow D^{* *} \ell \nu_{\ell}$. The branching ratio of each $B \rightarrow D^{* *} \ell \nu_{\ell}$ decay is varied within their uncertainties. The uncertainties are assumed to be $\pm 6 \%$ for $D_{1}, \pm 10 \%$ for $D_{2}^{*}, \pm 83 \%$ for $D_{1}^{\prime}$, and $\pm 100 \%$ for $D_{0}^{*}$, respectively, including limited knowledge of the $D^{* *}$ decays. Furthermore, we consider the impact of contributions from radially excited $D(2 S)$ and $D^{*}(2 S)$, where we consider the assuming branching ratios of $B \rightarrow D^{(*)}(2 S) \ell \nu_{\ell}$ to be as much as $0.5 \%$ each. The yields of fake $D^{*}$ events are fixed to the values estimated from sidebands in the $\Delta M$ distributions. We vary the fixed yields of fake $D^{(*)}$ events within the uncertainties. To take into account possible dependence of PDF shape to $D$ meson sub-decay mode, we vary the calibration factors for each $D$ meson sub-decay mode within their uncertainties for events with falsely reconstructed $D^{(*)}$ events. The yields of other background processes, predominantly from $B \rightarrow X_{c} D^{*}$ events, are fixed to the values estimated from MC simulation. We consider variations on the yield and shape of the PDF of these background processes, corresponding to their measured uncertainties. The uncertainties of each $B \rightarrow X_{c} D^{*}$ decays are assumed to be $\pm 8 \%$ for $B \rightarrow D_{s}^{*} D^{*-}, \pm 14 \%$ for $B \rightarrow D_{s} D^{*-}, \pm 8 \%$ for $B \rightarrow D^{*+} D^{*-}$, and $\pm 10 \%$ for $B \rightarrow D^{+} D^{*-}$, respectively. Furthermore, we add an uncertainty of $\pm 4 \%$ due to the size of the MC sample. We include an uncertainty on the branching ratio of $D_{s} \rightarrow \tau \nu_{\tau}$ decay, which may peak near the signal in the $E_{\mathrm{ECL}}$ distribution: it is found to be negligible. The reconstruction efficiency ratio of signal to normalization events is varied within its uncertainty, which is limited by the size of MC sam- ples for signal events. We include other minor systematic uncertainties from two sources. One is an uncertainty from the parameters that are used for the reweighting of the semileptonic $B \rightarrow D^{(*(*))} \ell \nu_{\ell}$ decays from the ISGW model to the LLSW model. The other is an uncertainty on the branching ratio of $\tau^{-} \rightarrow \ell^{-} \bar{\nu}_{\ell} \nu_{\tau}$ decay [20]. The total systematic uncertainty is estimated by summing the above uncertainties in quadrature.

\section{RESULTS}

The projection of the fitted distributions are shown in Figure 1]. The yields of signal and normalization events are measured to be $231 \pm 23$ (stat) and $2800 \pm 57$ (stat), respectively. The ratio $\mathcal{R}\left(D^{*}\right)$ is therefore found to be

$$
\mathcal{R}\left(D^{*}\right)=0.302 \pm 0.030 \pm 0.011
$$

where the first and second errors correspond to statistical and systematic uncertainties, respectively.

We calculate the statistical significance of the signal as $\sqrt{-2 \ln \left(\mathcal{L}_{0} / \mathcal{L}_{\text {max }}\right)}$, where $\mathcal{L}_{\text {max }}$ and $\mathcal{L}_{0}$ are the maximum likelihood and the likelihood obtained assuming zero signal yield, respectively. We obtain a statistical significance of $13.8 \sigma$. We also estimate the compatibility of the measured value of $\mathcal{R}\left(D^{*}\right)$ and the SM prediction. The effect of systematic uncertainties are included by convolving the likelihood function with a Gaussian distribution. We obtain that our result is larger than the SM prediction by $1.6 \sigma$.

\section{CROSS-CHECKS}

To determine the consistency among $\tau$ final states, we divide the data samples by lepton flavor on the signal side and fit them separately. The efficiency ratios $\varepsilon_{\text {norm }} / \varepsilon_{\text {sig }}$ are estimated to be $1.107 \pm 0.016$ and $1.591 \pm 0.030$ for electron and muon channels of the tau decays, respectively. We obtain

$$
\begin{aligned}
& \mathcal{R}\left(D^{*}\right)=0.311 \pm 0.038 \pm 0.013\left(\ell^{\text {sig }}=e\right), \\
& \mathcal{R}\left(D^{*}\right)=0.304 \pm 0.051 \pm 0.018\left(\ell^{\operatorname{sig}}=\mu\right),
\end{aligned}
$$

where the first and second errors correspond to statistical and systematic uncertainties, respectively. The systematic uncertainties are summarized in Table \. These two results are found to be consistent with each other.

To study $B \rightarrow D^{* *} \ell \nu_{\ell}$ background contributions, we require an additional $\pi^{0}$ in addition to the nominal event selection. In this control sample, we calculate $E_{\mathrm{ECL}}^{\prime}$, which is defined as the remaining energy after the energy deposit from the additional $\pi^{0}$ is removed from $E_{\mathrm{ECL}}$. The $B \rightarrow D^{* *} \ell \nu_{\ell}$ background contributions are extracted from them control samples using the nominal fitting method, replacing $E_{\mathrm{ECL}}$ with $E_{\mathrm{ECL}}^{\prime}$. We found consistent results for the branching ratios of $B \rightarrow D^{* *} \ell \nu_{\ell}$ in the control and signal regions. 
TABLE I. List of relative systematic uncertainties in percent.

\begin{tabular}{c|c|c|c}
\hline & \multicolumn{3}{|c}{$\mathcal{R}\left(D^{*}\right)[\%]$} \\
\hline Sources & $\ell^{\text {sig }}=e, \mu$ & $\ell^{\text {sig }}=e$ & $\ell^{\text {sig }}=\mu$ \\
\hline MC statistics for each PDF shape & $2.2 \%$ & $2.5 \%$ & $3.9 \%$ \\
PDF shape of the normalization in cos $\theta_{B-D^{*} \ell}$ & ${ }_{-0.0}^{+1.1} \%$ & ${ }_{-0.0}^{+2.1} \%$ & ${ }_{-0.0}^{+2.8} \%$ \\
PDF shape of $B \rightarrow D^{* *} \ell \nu_{\ell}$ & ${ }_{-1.7}^{+1.0} \%$ & ${ }_{-1.3}^{+0.7} \%$ & ${ }_{-3.3}^{+2.2} \%$ \\
PDF shape and yields of fake $D^{(*)}$ & $1.4 \%$ & $1.6 \%$ & $1.6 \%$ \\
PDF shape and yields of $B \rightarrow X_{c} D^{*}$ & $1.1 \%$ & $1.2 \%$ & $1.1 \%$ \\
Reconstruction efficiency ratio $\varepsilon_{\text {norm }} / \varepsilon_{\text {sig }}$ & $1.2 \%$ & $1.5 \%$ & $1.9 \%$ \\
Modeling of semileptonic decay & $0.2 \%$ & $0.2 \%$ & $0.3 \%$ \\
$\mathcal{B}\left(\tau^{-} \rightarrow \ell^{-} \bar{\nu}_{\ell} \nu_{\tau}\right)$ & $0.2 \%$ & $0.2 \%$ & $0.2 \%$ \\
\hline Total systematic uncertainties & ${ }_{-3.5}^{+3.4} \%$ & ${ }_{-3.7}^{+4.1} \%$ & ${ }_{-5.8}^{+5.9} \%$ \\
\hline
\end{tabular}

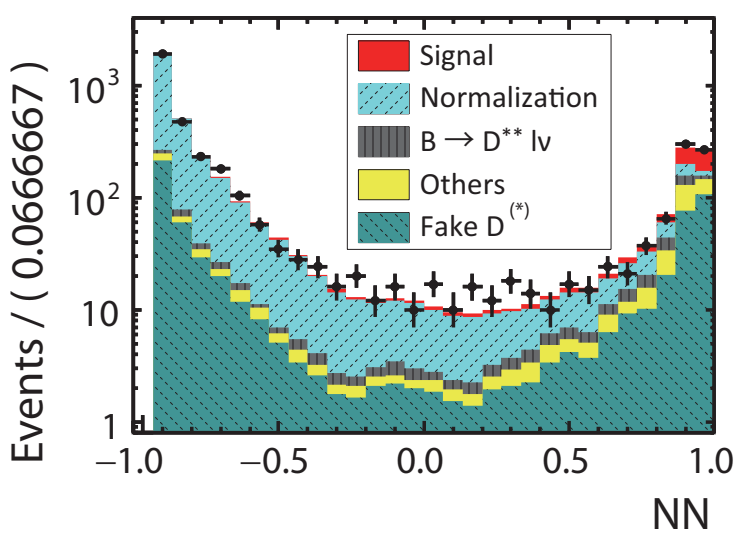

(a) $N N$ distribution

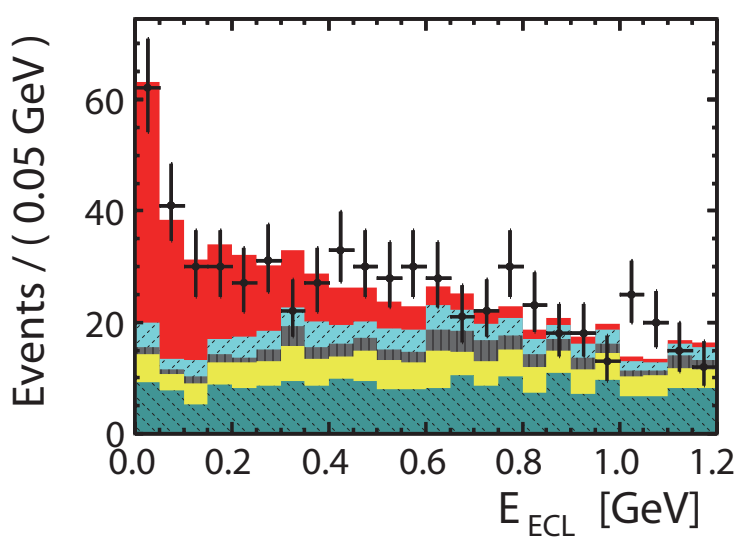

(b) $E_{\mathrm{ECL}}$ distribution with signal-enhanced $N N$ region $(N N>0.8)$

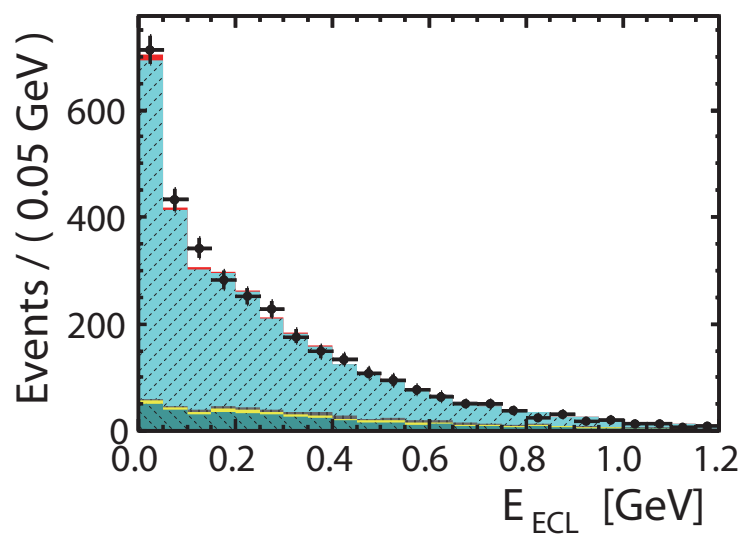

(c) $E_{\mathrm{ECL}}$ distribution with normalization-enhanced $N N$ region $(N N<0.8)$

FIG. 1. Projections of the fit results with data points overlaid. The background categories are described in detail in the text, where "others" refers to predominantly $B \rightarrow X_{c} D^{*}$ decays.

\section{NEW PHYSICS COMPATIBILITY TESTS}

We investigated the compatibility of the data samples with type II two-Higgs-doublet model (2HDM) and lep- toquark models. Assuming all neutrinos are left-handed, the effective Hamiltonian that contains all possible fourfermion operators for the $b \rightarrow c \tau \nu_{\tau}$ decay can be de- 
scribed as follows:

$$
\mathcal{H}_{\text {eff }}=\frac{4 G_{F}}{\sqrt{2}} V_{c b}\left[\mathcal{O}_{V_{1}}+\sum_{X=S_{1}, S_{2}, V_{1}, V_{2}, T} C_{X} \mathcal{O}_{X}\right],
$$

where the four-Fermi operators, $\mathcal{O}_{X}$, are defined as

$$
\begin{aligned}
\mathcal{O}_{S_{1}} & =\left(\bar{c}_{L} b_{R}\right)\left(\bar{\tau}_{R} \nu_{\tau L}\right), \\
\mathcal{O}_{S_{2}} & =\left(\bar{c}_{R} b_{L}\right)\left(\bar{\tau}_{R} \nu_{\tau L}\right), \\
\mathcal{O}_{V_{1}} & =\left(\bar{c}_{L} \gamma^{\mu} b_{L}\right)\left(\bar{\tau}_{L} \gamma_{\mu} \nu_{\tau L}\right), \\
\mathcal{O}_{V_{2}} & =\left(\bar{c}_{R} \gamma^{\mu} b_{R}\right)\left(\bar{\tau}_{L} \gamma_{\mu} \nu_{\tau L}\right), \\
\mathcal{O}_{T} & =\left(\bar{c}_{R} \sigma^{\mu \nu} b_{L}\right)\left(\bar{\tau}_{R} \sigma_{\mu \nu} \nu_{\tau L}\right),
\end{aligned}
$$

and the $C_{X}$ parameters correspond to the Wilson coefficients of $\mathcal{O}_{X}$. In the type II $2 \mathrm{HDM}$, the relevant Wilson coefficient is given as $C_{S_{1}}=-m_{b} m_{\tau} \tan ^{2} \beta / m_{H^{+}}^{2}$, where $\tan \beta$ is the ratio of the vacuum expectation values of the two Higgs doublets, and $m_{b}, m_{\tau}$, and $m_{H^{+}}$are the masses of the $b$ quark, $\tau$ lepton, and charged Higgs boson, respectively. In $\bar{B}^{0} \rightarrow D^{*+} \tau^{-} \bar{\nu}_{\tau}$ decay, the influence by $\mathcal{O}_{S_{2}}$ operator is identical with that by $\mathcal{O}_{S_{1}}$ except for the opposite sign of corresponding Wilson coefficient [16]. If we consider a contribution from $\mathcal{O}_{V_{1}, V 2}$ by a new vector boson $W^{\prime}$, which couples to left- or right-handed fermion currents, we must seriously take tight constraints by the ATLAS 24, 25] and CMS [26, 27] experiments at the LHC. Various leptoquark models have been presented to explain anomalies on $\mathcal{R}\left(D^{(*)}\right)$ in Ref. 28]. Some of leptoquark models generate the tensor operator, which is the most sensitive operator to $B \rightarrow D^{*} \tau \nu_{\tau}$ decay. We choose one representative model, denoted $R_{2}$, as a benchmark, which contains a scalar leptoquark with quantum numbers $\left(S U(3)_{c}, S U(2)_{L}\right)_{Y}=(3,2)_{7 / 6}$, where $S U(3)_{c}, S U(2)_{L}, Y$ are the QCD representation, the weak isospin representation, and the hypercharge, respectively. In this leptoquark model, the relevant Wilson coefficients are related by $C_{S_{2}}=+7.8 C_{T}$ at the $b$ quark mass scale, assuming a leptoquark mass scale of $1 \mathrm{TeV} . R_{2}$ type leptoquark model is dedicatedly discussed in Ref. [29], because it seems difficult to implement light vector leptoquarks in realistic scenarios and other types of scalar leptoquark models destabilize proton [30].

To determine the sensitivity to these models, we construct PDFs for signal events by scanning through values of $\tan \beta / m_{H^{+}}$in the type II $2 \mathrm{HDM}$, and $C_{T}$ in the $R_{2}$ type leptoquark model. For the former, $\tan \beta / m_{H^{+}}$is scanned from 0.0 to $1.0 \mathrm{GeV}^{-1}$ and for the latter $C_{T}$ is scanned from -0.150 to +0.400 , where we assume the Wilson coefficient to be real. Figure 2 and 3 demonstrates the dependence of the efficiency and measured values of $\mathcal{R}\left(D^{*}\right)$ on the values of the respective parameters in the type II $2 \mathrm{HDM}$ or the $R_{2}$ type leptoquark models. In the type II $2 \mathrm{HDM}$, the efficiency drops by as much as $5 \%$ for large values of $\tan \beta / m_{H^{+}}$, mainly due to the variation of the lepton momentum distribution. On the other hand, in the $R_{2}$ type leptoquark model, the efficiency increases by up to $16 \%$ at most, mainly due to the variation of the $D^{*}$ momentum distribution. The measured value of
$\mathcal{R}\left(D^{*}\right)$ matches the theoretical predictions in the type II $2 \mathrm{HDM}$ around $\tan \beta / m_{H^{+}}=0.7 \mathrm{GeV}^{-1}$, while the measured value of $\mathcal{R}\left(D^{*}\right)$ matches the theoretical predictions in the $R_{2}$ type leptoquark model at two points: $C_{T}=-0.03$ and +0.36 .

In Refs. 7] and [4], the $q^{2} \equiv\left(p_{B}-p_{D^{*}}\right)^{2}$ spectra are examined in order to study the effects of new physics beyond the SM. Since $q^{2}$ can not be calculated in this study due to the neutrino from the $B_{\mathrm{tag}}$, we use the momenta of the $D^{*}$ and the $\ell$ at $B_{\text {sig }}$ in $\Upsilon(4 S)$ rest frame instead of $q^{2}$. Figure 4 shows the momentum distributions of the background subtracted data for the SM, type II $2 \mathrm{HDM}$ with $\tan \beta / m_{H^{+}}=0.7 \mathrm{GeV}^{-1}$, and the $R_{2}$ type leptoquark model with $C_{T}=+0.36$. Table $\amalg$ shows $p$ values for the three scenarios, where we include only the statistical uncertainty. We find our data is compatible with the SM and type II $2 \mathrm{HDM}$ with $\tan \beta / m_{H^{+}}=0.7 \mathrm{GeV}^{-1}$, while the $R_{2}$ type leptoquark model with $C_{T}=+0.36$ is disfavored.

TABLE II. $p$ values for three scenarios.

\begin{tabular}{c|c|c|c}
\hline \hline & & \multicolumn{2}{|c}{$p$ values } \\
\cline { 3 - 4 } Model & Parameters & $p_{D^{*}}$ & $p_{\ell}$ \\
\hline SM & & $37.6 \%$ & $25.8 \%$ \\
Type II 2HDM & $\tan \beta / m_{H^{+}}=0.7 \mathrm{GeV}^{-1}$ & $37.9 \%$ & $22.5 \%$ \\
$R_{2}$ leptoquark model & $C_{T}=+0.36$ & $1.4 \%$ & $16.2 \%$ \\
\hline \hline
\end{tabular}

\section{CONCLUSION}

In conclusion, we report the first measurement of $\mathcal{R}\left(D^{*}\right)$ with a semileptonic tagging method using a data sample containing $772 \times 10^{6} B \bar{B}$ pairs collected with the Belle detector. The results are

$$
\mathcal{R}\left(D^{*}\right)=0.302 \pm 0.030 \text { (stat) } \pm 0.011 \text { (syst), }
$$

which is within $1.6 \sigma$ of the SM prediction including systematic uncertainties, is in good agreement with other measurements by Belle [5 7], BABAR [4], and LHCb [8] collaborations, and is statistically independent of earlier Belle measurements. We investigate the compatibility of the data samples with the type II $2 \mathrm{HDM}$ and the $R_{2}$ type leptoquark model. We find the most favored parameter points are around $\tan \beta / m_{H^{+}}=0.7 \mathrm{GeV}^{-1}$ in the type II $2 \mathrm{HDM}$ and $C_{T}=-0.030$ and +0.360 in the $R_{2}$ type leptoquark model, although the latter is disfavored when considering the impact on the decay kinematics.

\section{ACKNOWLEDGEMENTS}

We thank Y. Sakaki, R. Watanabe, and M. Tanaka for their invaluable suggestions. This work was supported in 


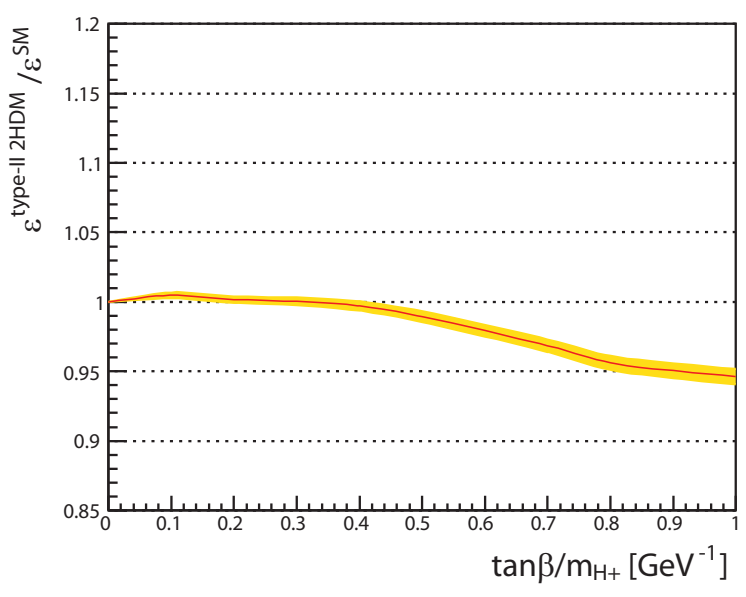

(a)Type II 2HDM.

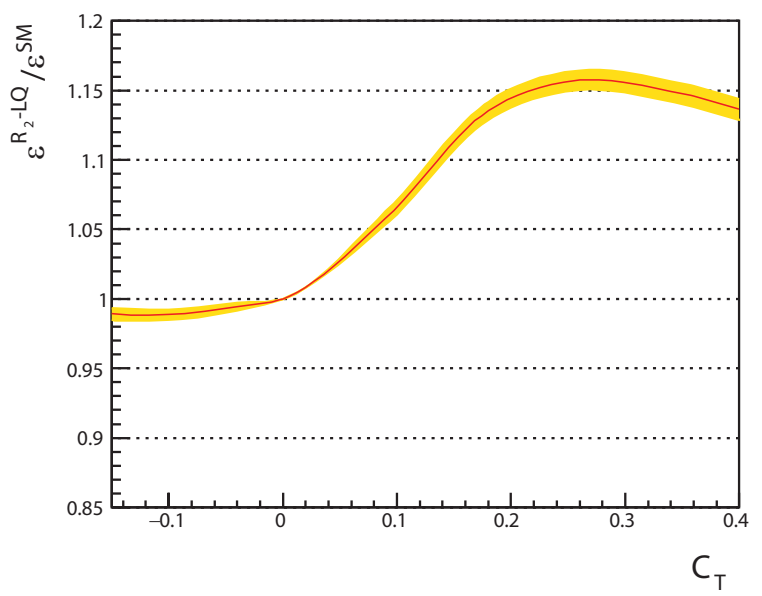

(b) $R_{2}$ type leptoquark model.

FIG. 2. Efficiency in (a) type II $2 \mathrm{HDM}$ and (b) $R_{2}$ type leptoquark model with respect to the SM value.

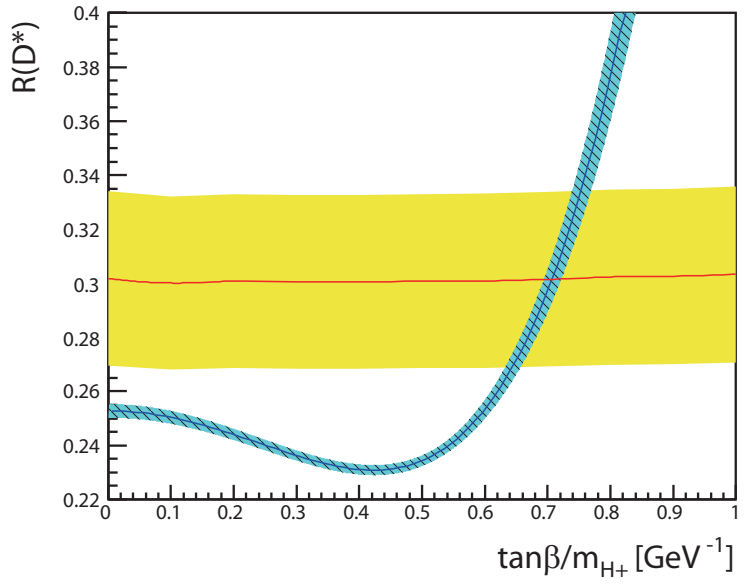

(a)Type II 2HDM.

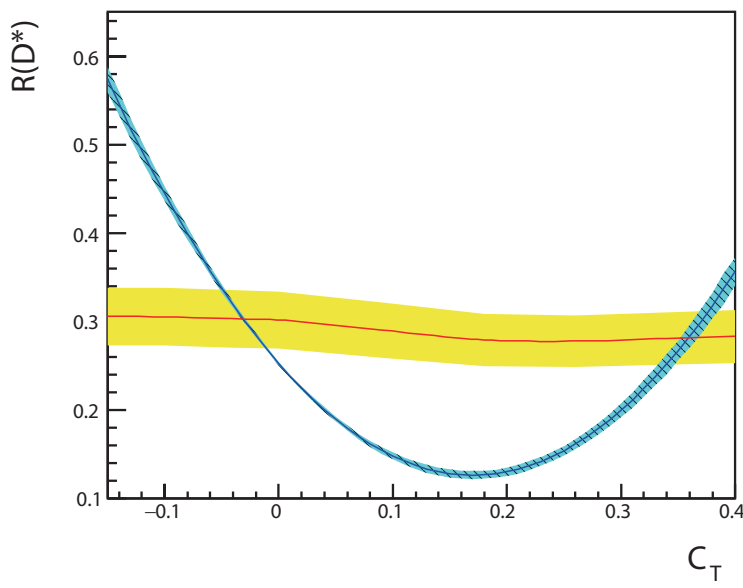

(b) $R_{2}$ type leptoquark model.

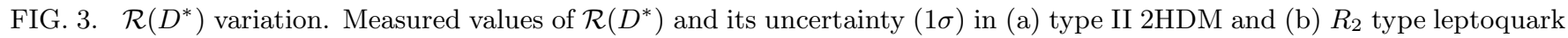
model are shown by solid curve (red) and shaded region (yellow). Theoretical prediction and its uncertainty $(1 \sigma)$ are shown by solid curve (blue) and hatched region (light blue) [16].

part by a Grant-in-Aid for JSPS Fellows (No.13J03438) and a Grant-in-Aid for Scientific Research (S) "Probing New Physics with Tau-Lepton" (No.26220706) We thank the KEKB group for the excellent operation of the accelerator; the KEK cryogenics group for the efficient operation of the solenoid; and the KEK computer group, the National Institute of Informatics, and the PNNL/EMSL computing group for valuable computing and SINET4 network support. We acknowledge support from the Ministry of Education, Culture, Sports, Science, and Technology (MEXT) of Japan, the Japan Society for the Promotion of Science (JSPS), and the Tau-Lepton Physics Research Center of Nagoya University; the Australian Research Council; Austrian
Science Fund under Grant No. P 22742-N16 and P 26794-N20; the National Natural Science Foundation of China under Contracts No. 10575109, No. 10775142, No. 10875115, No. 11175187, No. 11475187 and No. 11575017; the Chinese Academy of Science Center for Excellence in Particle Physics; the Ministry of Education, Youth and Sports of the Czech Republic under Contract No. LG14034; the Carl Zeiss Foundation, the Deutsche Forschungsgemeinschaft, the Excellence Cluster Universe, and the VolkswagenStiftung; the Department of Science and Technology of India; the Istituto Nazionale di Fisica Nucleare of Italy; the WCU program of the Ministry of Education, National Research Foundation (NRF) of Korea Grants No. 2011- 


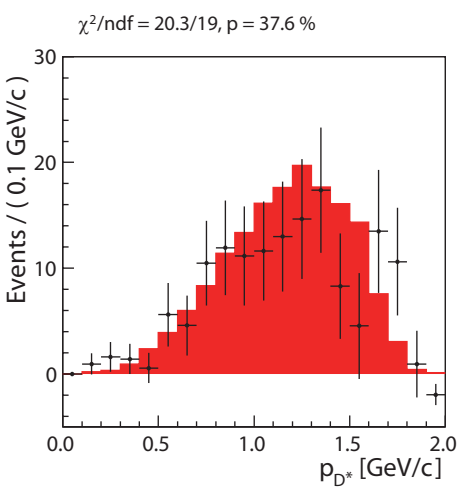

(a)SM.

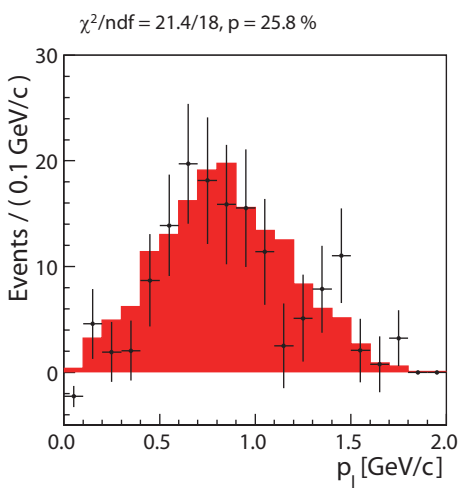

(d)SM.

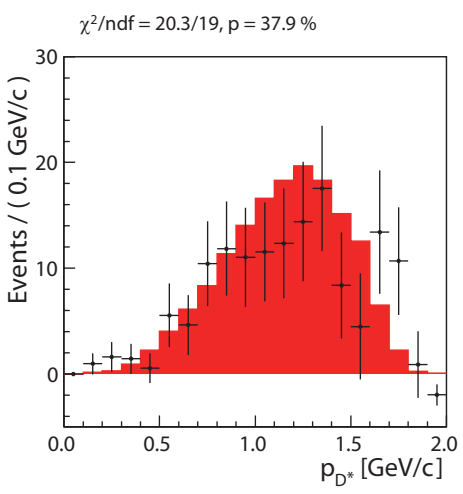

(b)Type II 2HDM with $\tan \beta / m_{H^{+}}=0.7 \mathrm{GeV}^{-1}$.

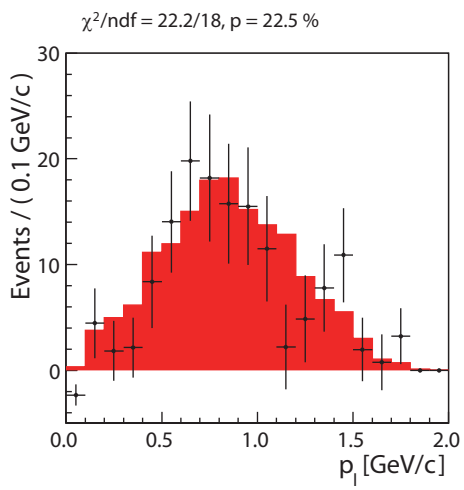

(e)Type II 2HDM with $\tan \beta / m_{H^{+}}=0.7 \mathrm{GeV}^{-1}$.

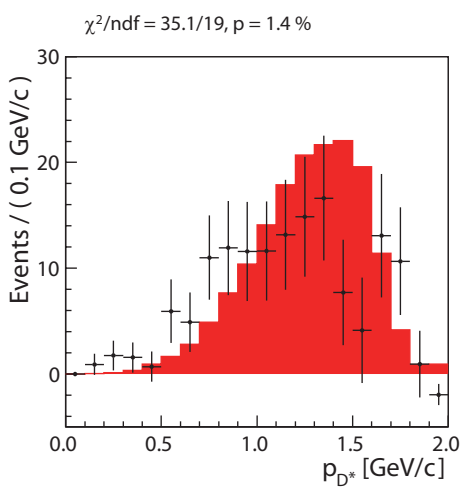

(c) $R_{2}$ type leptoquark model with $C_{T}=+0.36$.

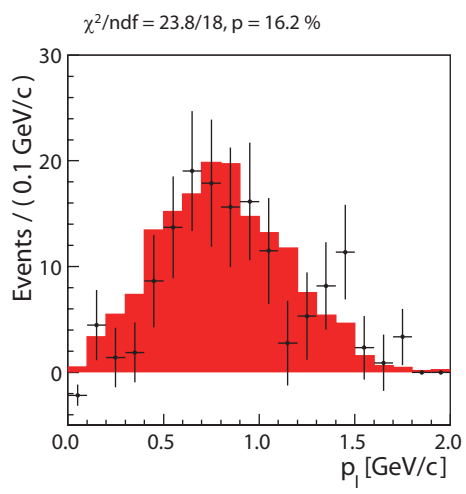

(f) $R_{2}$ type leptoquark model with $C_{T}=+0.36$

FIG. 4. Background-subtracted momenta distributions of $D^{*}$ (top) and $\ell$ (bottom) in the region of $N N>0.8$ and $E_{\mathrm{ECL}}<0.5$ $\mathrm{GeV}$. The points and the shaded histograms correspond to the measured and expected distributions, respectively. The expected distributions are normalized to the number of detected events.

0029457, No. 2012-0008143, No. 2012R1A1A2008330, No. 2013R1A1A3007772, No. 2014R1A2A2A01005286, No. 2014R1A2A2A01002734, No. 2015R1A2A2A01003280, No. 2015H1A2A1033649; the Basic Research Lab program under NRF Grant No. KRF-2011-0020333, Center for Korean J-PARC Users, No. NRF-2013K1A3A7A06056592; the Brain Korea 21-Plus program and Radiation Science Research Institute; the Polish Ministry of Science and Higher Education and the National Science Center; the Ministry of Education and Science of the Russian
Federation and the Russian Foundation for Basic Research; the Slovenian Research Agency; Ikerbasque, Basque Foundation for Science and the Euskal Herriko Unibertsitatea (UPV/EHU) under program UFI 11/55 (Spain); the Swiss National Science Foundation; the Ministry of Education and the Ministry of Science and Technology of Taiwan; and the U.S. Department of Energy and the National Science Foundation. This work is supported by a Grant-in-Aid from MEXT for Science Research in a Priority Area ("New Development of Flavor Physics") and from JSPS for Creative Scientific Research ("Evolution of Tau-lepton Physics").
[1] Charge-conjugate decays are implied throughout this paper, unless otherwise stated.

[2] S. Fajfer, J.F.Kamenik, and I. Nisandzic, Phys. Rev. D 85, 094025 (2012).

[3] J.F. Kamenik, and F. Mescia, Phys. Rev. D 78, 014003 (2008).

[4] J.P. Lees et al. (BABAR Collaboration), Phys. Rev. Lett. 109, 101802 (2012); J.P. Lees et al. (BABAR Collaboration), Phys. Rev. D 88, 072012 (2013);
[5] A. Matyja et al. (Belle Collaboration), Phys. Rev. Lett. 99, 191807 (2007).

[6] A. Bozek et al. (Belle Collaboration), Phys. Rev. D 82, 072005 (2010).

[7] M. Huschle et al. (Belle Collaboration), Phys. Rev. D 92, 072014 (2015).

[8] R. Aaij et al. (LHCb Collaboration), Phys. Rev. Lett. 115, 111803 (2015). 
[9] Y. Amhis et al., arXiv:1412.7515 and online update at http://www.slac.stanford.edu/xorg/hfag/

[10] B. Kronenbitter et al. (Belle Collaboration), Phys. Rev. D 92, 051102(R) (2015).

[11] B. Aubert et al. (BABAR Collaboration), Phys. Rev. D 81, 051101(R) (2010).

[12] A. Abashian et al. (Belle Collaboration), Nucl. Instrum. Methods Phys. Res., Sect. A 479, 117 (2002); also see the detector section in J. Brodzicka et al., Prog. Theor. Exp. Phys. (2012) 04D001.

[13] S. Kurokawa and E. Kikutani, Nucl. Instrum. Methods Phys. Res., Sect. A 499, 1 (2003), and other papers included in this volume; T. Abe et al., Prog. Theor. Exp. Phys. (2013) 03A001 and following articles up to 03A011.

[14] D.J. Lange Nucl. Instrum. Methods Phys. Res., Sect. A 462, 152 (2001).

[15] R. Brun et al., GEAN3.21, CERN Report No. DD/EE/84-1, 1984 (unpublished)

[16] M. Tanaka and R. Watanabe, Phys. Rev. D 87, 034028 (2013).

[17] D. Scora and N. Isgur, Phys. Rev. D 52, 2783 (1995).

[18] A.K. Leibovich, Z. Ligeti, I.W. Stewart, and M.B. Wise, Phys. Rev. D 57, 308 (1998).
[19] E. Nakano, Nucl. Instrum. Methods Phys. Res., Sect. A 494, 402 (2002).

[20] Particle Data Group, Chin. Phys. C 38, 090001 (2014).

[21] M. Feindt and U. Kerzel, Nucl. Instrum. Methods Phys. Res., Sect. A 559, 190 (2006).

[22] T. Skwarnicki, Ph.D. Thesis, Institute for Nuclear Physics, Krakow 1986; DESY Internal Report, DESY F31-86-02 (1986).

[23] J.H. Friedman, Data Analysis Techniques for High Energy Particle Physics, in: Proc. 1974 CERN School of Computing, CERN 74-23 (1974).

[24] ATLAS Collaboration, Eur. Phys. J. C 75, 165 (2015)

[25] ATLAS Collaboration, Phys. Lett. B 743, 235 (2015)

[26] CMS Collaboration, Phys. Lett. B 718, 1229 (2013)

[27] CMS Collaboration, arXiv:1508.04308

[28] Y. Sakaki, R. Watanabe, M. Tanaka, and A. Tayduganov, Phys. Rev. D 88, 094012 (2013).

[29] I. Doršner, S. Fajfer, N. Košnik, and I. Nišandžić, JHEP, 11, 084 (2013).

[30] I. Doršner, S. Fajfer, and N. Košnik, Phys. Rev. D 86, 015013 (2012). 